Götz, Kristina; Kutzelmann, Sabine

\title{
Leseflüssigkeit und Lesemotivation: Die beiden Förderdimensionen des
}

\section{Mehrsprachigen Lesetheaters}

Mehrsprachiges Lesetheater. Handbuch zu Theorie und Praxis. Opladen; Berlin; Toronto : Verlag Barbara Budrich 2017, S. 57-69

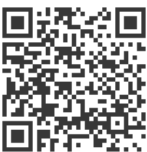

Quellenangabe/ Reference:

Götz, Kristina; Kutzelmann, Sabine: Leseflüssigkeit und Lesemotivation: Die beiden

Förderdimensionen des Mehrsprachigen Lesetheaters - In: Mehrsprachiges Lesetheater. Handbuch zu

Theorie und Praxis. Opladen; Berlin; Toronto : Verlag Barbara Budrich 2017, S. 57-69 - URN:

urn:nbn:de:0111-pedocs-209806 - DOI: 10.25656/01:20980

https://nbn-resolving.org/urn:nbn:de:0111-pedocs-209806

https://doi.org/10.25656/01:20980

in Kooperation mit / in cooperation with:

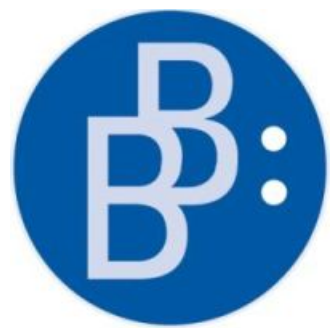

https://www.budrich.de

\section{Nutzungsbedingungen}

Gewährt wird ein nicht exklusives, nicht übertragbares, persönliches und beschränktes Recht auf Nutzung dieses Dokuments. Dieses Dokument ist ausschließlich für den persönlichen, nicht-kommerziellen Gebrauch bestimmt. Die Nutzung stellt keine Übertragung des Eigentumsrechts an diesem Dokument dar und gilt vorbehaltlich der folgenden Einschränkungen: Auf sämtlichen Kopien dieses Dokuments müssen alle Urheberrechtshinweise und sonstigen Hinweise auf gesetzlichen Schutz beibehalten werden. Sie dürfen dieses Dokument nicht in irgendeiner Weise abändern, noch dürfen Sie dieses Dokument für öffentliche oder kommerzielle Zwecke vervielfältigen, öffentlich ausstellen, aufführen, vertreiben oder anderweitig nutzen.

Mit der Verwendung dieses Dokuments erkennen Sie die Nutzungsbedingungen an.

\section{Kontakt / Contact:}

peDOCS

DIPF | Leibniz-Institut für Bildungsforschung und Bildungsinformation Informationszentrum (IZ) Bildung

E-Mail: pedocs@dipf.de

Internet: www.pedocs.de

\section{Terms of use}

We grant a non-exclusive, non-transferable, individual and limited right to using this document.

This document is solely intended for your personal, non-commercial use. Use of this document does not include any transfer of property rights and it is conditional to the following limitations: All of the copies of this documents must retain all copyright information and other information regarding legal protection. You are not allowed to alter this document in any way, to copy it for public or commercial purposes, to exhibit the document in public, to perform, distribute or otherwise use the document in public.

By using this particular document, you accept the above-stated conditions of use. 
Sabine Kutzelmann Ute Massler Klaus Peter Kristina Götz Angelika Ilg (Hrsg.)

\section{Mehrsprachiges Lesetheater}

Handbuch zu Theorie und Praxis 
Mehrsprachiges Lesetheater 

Sabine Kutzelmann • Ute Massler • Klaus Peter • Kristina Götz • Angelika Ilg (Hrsg.)

\section{Mehrsprachiges Lesetheater}

Handbuch zu Theorie und Praxis

Verlag Barbara Budrich

Opladen • Berlin • Toronto 2017 
Bibliografische Information der Deutschen Nationalbibliothek

Die Deutsche Nationalbibliothek verzeichnet diese Publikation in der Deutschen Nationalbibliografie; detaillierte bibliografische Daten sind im Internet über http://dnb.d-nb.de abrufbar.

Gefördert durch

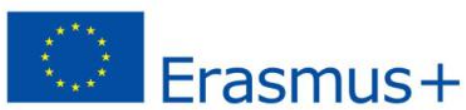

Schulbildung

Dieses Projekt wurde mit Unterstützung der Europäischen Kommission finanziert. Die

Verantwortung für den Inhalt dieser Veröffentlichung (Mitteilung) trägt allein der Verfasser; die Kommission haftet nicht für die weitere Verwendung der darin enthaltenen Angaben.

This project has been funded with support from the European Commission. This publication reflects the views only of the author, and the Commission cannot be held responsible for any use which may be made of the information contained therein.

Gedruckt auf säurefreiem und alterungsbeständigem Papier.

Alle Rechte vorbehalten.

C 2017 Verlag Barbara Budrich, Opladen, Berlin \& Toronto

www.budrich-verlag.de

$$
\text { ISBN 978-3-8474-2021-4 }
$$

eISBN 978-3-8474-1109-3

Das Werk einschließlich aller seiner Teile ist urheberrechtlich geschützt. Jede Verwertung außerhalb der engen Grenzen des Urheberrechtsgesetzes ist ohne Zustimmung des Verlages unzulässig und strafbar. Das gilt insbesondere für Vervielfältigungen, Übersetzungen, Mikroverfilmungen und die Einspeicherung und Verarbeitung in elektronischen Systemen.

Umschlaggestaltung: Bettina Lehfeldt, Kleinmachnow - www.lehfeldtgraphic.de

Lektorat und Satz: Ulrike Weingärtner, Gründau

Titelbildnachweis: Stillaufnahme aus dem MELT Film „LOUD, DEUTLICH, LENTEMENT: Mit

Mehrsprachigem Lesetheater Sprachgrenzen überwinden“ (s. Projektwebsite: http://melt-multilingualreaders-theatre.eu/ )

Druck: paper \& tinta, Warschau

Printed in Europe 


\section{Inhalt}

Das Projekt Mehrsprachiges Lesetheater: Ausgangslage, Zielsetzung

und praxisrelevante Ergebnisse der Entwicklung .................

Sabine Kutzelmann, Ute Massler und Robert Hendel

\section{Teil A}

\section{Forschungsmethodik}

1 Design-Based Research: Eine Forschungsstrategie zur Entwicklung nachhaltiger Innovationen in der schulischen Praxis . . . . . . . . . . . . Sabine Kutzelmann und Kristina Götz

2 Die schulischen Erprobungskontexte des MELT-Projekts . . . . . . . . . . . 32 Kristina Götz, Robert Hendel, Sabine Kutzelmann und Klaus Peter

3 Mehrsprachiges Lesetheater: Ergebnisse zur Akzeptanz dieser

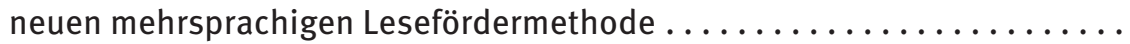
Kristina Götz, Sabine Kutzelmann und Ute Massler

Teil B

Theoretische Grundlagen des

Entwicklungs- und Forschungsprozesses

1 Leseflüssigkeit und Lesemotivation: Die beiden Förderdimensionen des Mehrsprachigen Lesetheaters . . . . . . . . . . . . . . . Kristina Götz und Sabine Kutzelmann

2 Das Unterrichtsdesign Mehrsprachiges Lesetheater aus linguistischer Sicht.

Klaus Peter und Dominik Unterthiner

3 Vom Readers' Theatre über interlinguale Literatur zum Mehrsprachigen Lesetheater: Die didaktisch-methodischen Leitlinien der Gestaltung im Kontext der Mehrsprachigkeitsdidaktik ................. Ute Massler und Sabine Kutzelmann

\section{Teil C}

\section{Das didaktisch-methodische Design:}

\section{Theoretische Grundlagen und Anleitung für die Praxis}

1 Mehrsprachige Lesetheaterstücke: Konzeption der Lehr-Lern-Materialien . . . Angelika Ilg, Franz Ludescher und Seraina Paul

2 Mehrsprachiges Vorlesen durch die Lehrperson als Design-Komponente des Mehrsprachigen Lesetheaters . . . . . . . . . . . . . . . . . . 
3 Miteinander sind alle Stars - Die Bedeutung des kooperativen Lernens

im Mehrsprachigen Lesetheater.

120

Sabine Kutzelmann und Kerstin Theinert

4 Literarisches Lernen als Design-Komponente des

Mehrsprachigen Lesetheaters

Angelika Ilg und Kerstin Theinert

5 Die zentralen Lehr-Lern-Prozesse des Mehrsprachigen Lesetheaters:

Eine Anleitung für die Praxis

Sabine Kutzelmann, Ute Massler, Klaus Peter unter Mitarbeit von

Anne-Marie Antony, Stefanie Arnold, Kurt Benedikt, Andrea Büchel,

Stefanie Blodnig, Tessy Gollère, Robert Hendel und Christian Mertens

\section{Teil D}

\section{Curriculum Lehrerfortbildung}

1 Professionswissen und Einstellungen von Sprachlehrpersonen zur

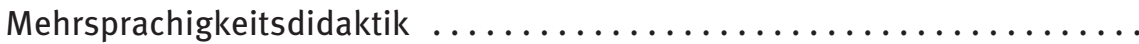
Klaus Peter und Dominik Unterthiner

2 Sprach- und Fachgrenzen überwinden: Die didaktische und strategische Konzeption des Fortbildungscurriculums zur Lesefördermethode

Mehrsprachiges Lesetheater ......................... 168

Sabine Kutzelmann und Ute Massler

Anhang 1) Anleitung zur Dramatisierung von mehrsprachigen Lesetheatern am Beispiel von Bend it like Beckham . . . . . . . . . . . . . . . . . 181 Anhang 2) Lesetheater Heidi . . . . . . . . . . . . . . . . . . . . . . 185

Anhang 3) Lesetheater Luina - Die Seejungfrau vom Bodensee . . . . . . . . . . . 189

Anhang 4) Lesetheater Nasreddin Hodscha . . . . . . . . . . . . . . . . . . . . 191

Anhang 5) Lesetheater Dr Jekyll and Mr Hyde . . . . . . . . . . . . . . . . . 195

Anhang 6) Toolbox: Dramapädagogik ...................... 196

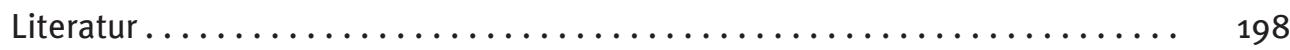

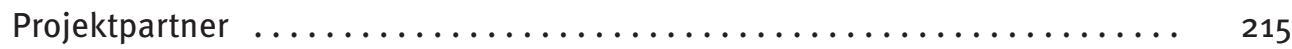

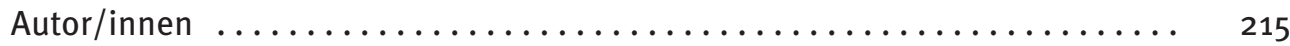




\title{
Teil B \\ Theoretische Grundlagen des \\ Entwicklungs- und Forschungsprozesses
}

\section{Leseflüssigkeit und Lesemotivation: Die beiden Förderdimensionen des Mehrsprachigen Lesetheaters}

\author{
Kristina Götz und Sabine Kutzelmann
}

Dieses Kapitel stellt den Forschungsstand bezogen auf die beiden Förderdimensionen Leseflüssigkeit und Lesemotivation dar, der während des Entwicklungs- und Forschungsprozesses als theoretischer Bezugsrahmen diente. Es wird erläutert, wie die mehrsprachige Lesefördermethode MELT theoriegeleitet entwickelt wurde, sodass nicht nur kognitive, sondern auch motivationale Aspekte der Leseförderung umgesetzt werden konnten. Der mehrsprachige Ansatz soll zu einer umfassenden Lesefördermaßnahme führen, die sprachübergreifend im Schul- und Fremdsprachenunterricht eingesetzt werden kann. Dadurch können Synergien zwischen den Unterrichtsfächern optimal genutzt werden. Im Folgenden werden für jede der beiden Förderdimensionen die theoretischen Grundlagen beschrieben, die dem Designprozess der Maßnahme zugrunde lagen. Dabei wird jeweils auf die Bedeutung der Dimensionen sowohl in der Erst- als auch in der Fremdsprache eingegangen. Zunächst werden theoretische Überlegungen und empirische Befunde zur Leseflüssigkeit in der Erstsprache (1.1) und Fremdsprache (1.2) erläutert. Im Folgenden wird dargestellt, wie diese bei der Gestaltung der Maßnahme umgesetzt wurden (1.3). Dabei wird auch auf die Erfassung der Leseflüssigkeit eingegangen (1.3.1), mit der sich die Lehrperson einen Eindruck über die Lesekompetenz der Schüler/innen verschaffen kann. Dies ist wiederum für die Passung der Lernmaterialien, den mehrsprachigen Lesetheaterstücken, auf das sprachliche und kognitive Leistungsniveau der Klasse relevant. Danach werden in einem zweiten Teil die Desiderate der Forschung zur Lesemotivation in der Erstsprache (1.4) dargelegt, bevor wenige Befunde zur Bedeutung der Lesemotivation in der Fremdsprache besprochen werden (1.5). Abschließend wird dargestellt, wie diese Forschungsdesiderate bei der Gestaltung der motivationsfördernden Aspekte der Lesefördermethode MELT eingeflossen sind (1.6).

\subsection{Theoretische Grundlagen der Förderdimension Leseflüssigkeit}

Leseflüssigkeit ist ein mehrdimensionales Konstrukt, das vier Dimensionen umfasst (vgl. Rosebrock/Nix 2006): Die Lesegenauigkeit (exaktes De- bzw. Rekodieren von 
Wörtern), die Automatisierung der basalen Dekodierprozesse, die Lesegeschwindigkeit und die Prosodie. Um flüssig zu lesen, müssen Lesende die Wörter im Text akkurat und möglichst schnell (automatisch) erkennen bzw. dekodieren. Aus der Kombination dieser beiden Prozesse ergibt sich die Lesegeschwindigkeit. Eine integrale Dimension, die die Leseflüssigkeit von der reinen Lesegeschwindigkeit abgrenzt, ist die Prosodie, d. h. die Fähigkeit des ausdrucksstarken Vorlesens, zu der die sinnvolle Betonung, Intonation, Pausengestaltung sowie ein angemessener Rhythmus beim Lesen gehören (vgl. Rosebrock et al. 2016: 19). In der Forschungsliteratur wird bei der Darstellung der Leseflüssigkeit die Dimension des ausdrucksstarken Vorlesens allerdings meist wenig berücksichtigt; in der Regel wird Leseflüssigkeit lediglich durch die Anzahl der richtig gelesenen Wörter in einem bestimmten Zeitintervall operationalisiert (vgl. 1.4.). Dies lässt sich damit erklären, dass sinnerfassendes Lesen erst möglich wird, wenn eine gewisse Lesegeschwindigkeit vorhanden ist. Rosebrock et al. (2016) diskutieren Normwerte der Lesegeschwindigkeit und kommen zu einer Richtgröße von „nicht weniger als 100 Wörter[n] pro Minute bei nicht mehr als fünf Fehlern auf 100 Wörter" (Rosebrock et al. 2016: 64). Erst bei dieser Lesegeschwindigkeit und -genauigkeit kann man davon ausgehen, dass der Sinn des gelesenen Textes tatsächlich erfasst wird.

Das oberste Ziel des Lesens ist es, Information aus dem gelesenen Text zu ziehen und zu verarbeiten. Nach dem Ansatz des Simple View of Reading (Gough/Tunmer 1986) wird das Leseverständnis als die Summe aus der Dekodierfertigkeit und dem Sprachverständnis verstanden. Zusätzlich zeigen empirische Studien, dass die Leseflüssigkeit eine weitere Determinante des Leseverständnisses ist (vgl. Ennemoser et al. 2012; Kim et al. 2012; Klauda/Guthrie 2008; Kuhn/Stahl 2003). Das flüssige Lesen erlaubt es dem Lesenden, sich dem Verstehen des Textes zu widmen, ohne dass es zu Verzögerungen kommt, weil Aufmerksamkeit auf das Dekodieren der einzelnen Wörter gelenkt werden muss (National Reading Panel 2000; Rosebrock et al. 2016; Vellutino et al. 2004), weshalb Leseflüssigkeit auch als Brücke zum Leseverständnis bezeichnet wird (vgl. Rasinski 2004: 3). Zu Beginn des Leseerwerbs werden das Leseverständnis und die Leseflüssigkeit durch die Dekodierfertigkeit geregelt. Erfolgt das Dekodieren mit zunehmender Leseerfahrung automatischer und fehlerfreier, steigert sich die Lesegeschwindigkeit, die wiederum sinnerfassendes Lesen (für das dann auch Sprachverständnis vonnöten ist) ermöglicht. Das Leseverständnis ist nach dem Anfangsunterricht, wenn Schüler/innen basale Lesekompetenzen erworben haben, abhängig von der Leseflüssigkeit und den linguistischen Kompetenzen (vgl. Ennemoser et al. 2012). Bei Schüler/innen mit Leseschwierigkeiten bleibt der Zusammenhang zwischen Leseflüssigkeit und Leseverständnis bis in spätere Schuljahre bestehen (vgl. Müller et al. 2013; Torgesen et al. 2001). Bei typisch entwickelten Lesenden hingegen nimmt der Zusammenhang mit zunehmender Leseerfahrung und -praxis ab (vgl. Slocum et al. 1995; Yovanoff et al. 2005), während der Zusammenhang zwischen Sprach- und Leseverständnis zunimmt (vgl. Ennemoser et al. 2012; Marx/Jungmann 2000).

Im normalen Entwicklungsverlauf steigt die Leseflüssigkeit während der Grundschuljahre stetig an (vgl. Gebauer et al. 2012; Müller et al. 2013), d. h. dass die Kinder zunehmend flüssiger lesen. Bezogen auf das reine Lesetempo zeigt sich auch noch nach der Grundschulzeit, dass typische Leser/innen mit zunehmendem Alter und Bildungsgrad schneller lesen (vgl. Tischler et al. 2013). Unterschiede in der Leseflüssigkeit zeigen sich früh und bleiben leider weitgehend stabil (vgl. de Jong/van der Leij 2002; 
Landerl/Wimmer 2008). Langsame Leser/innen bleiben ohne Intervention langsam und können daher oftmals wenig Sinn aus dem Gelesenen ziehen. Dies führt zu einer wesentlichen Benachteiligung im (Schul-)Alltag. Die Förderung der Leseflüssigkeit, und damit einhergehend auch des Leseverständnisses, ist daher unabdingbar.

Zur Förderung der Leseflüssigkeit eignen sich diverse Lautleseverfahren, die in der Literatur gut dokumentiert sind (vgl. Grabe 2009; National Reading Panel 2000; Rosebrock/Nix 2006; Rosebrock et al. 2016). Empirische Befunde zeigen, dass mehrfaches, lautes Lesen von Texten die Leseflüssigkeit sowie das Textverständnis von Kindern in den Grundschuljahren verbessert (vgl. Kuhn/Stahl 2003; Müller et al. 2013). Dabei haben sich Verfahren, bei denen die Lesenden unterstützt werden (sogenanntes assisted reading) als besonders wirksam erwiesen (vgl. Rosebrock/Nix 2006). In einer Metaanalyse zur Wirksamkeit deutschsprachiger Förderansätze bei Lese-Rechtschreib-Störung folgern Ise et al. (2012: 130), dass Lautleseverfahren gegenüber symptom-spezifischen Interventionen wie z. B. dem Training der phonologischen Bewusstheit überlegen sind. Wichtig ist dabei, dass die Lautleseverfahren regelmäßig und über einen längeren Zeitraum hinweg angewandt werden. Eine Förderdauer von mindestens 20 Wochen zeigte sich in dieser Metaanalyse als sehr effektiv, während die Wirksamkeit von Studien mit kürzerer Dauer nicht statistisch belegt werden konnte. Allerdings war in vier von fünf Studien ein Lesetraining, das über nur sechs Wochen durchgeführt wurde, wirksamer als der Einsatz von Wahrnehmungs- und Funktionsübungen, die über einen deutlich längeren Zeitraum durchgeführt wurden. Bedeutsam für das Mehrsprachige Lesetheater ist auch der Befund, dass die Förderung durch Lehrkräfte zu größeren Effekten führte als Fördermaßnahmen, die von außenstehenden Personen angeleitet wurden.

In der Deutschdidaktik empfehlen Rosebrock et al. (2016: 126) ein Lautlesetraining über mindestens ein halbes, wenn nicht ein ganzes Schuljahr hinweg, mit 15- bis 20-minütigen Trainingseinheiten, die drei- bis fünfmal pro Woche in den Regelunterricht mit integriert werden. Auch im englischsprachigen Raum empfiehlt Rasinski (2003, zitiert in Grabe 2009: 305) tägliche Leseübungseinheiten à 15 bis 30 Minuten über einen Zeitraum von mindestens zwei Monaten (oder länger).

Zusammengenommen etablieren die oben genannten Befunde die Bedeutsamkeit der Leseflüssigkeit. Allerdings beziehen sich alle Befunde auf die Flüssigkeit, mit der Texte in der Erstsprache (im Folgenden L1) gelesen werden.

\subsection{Die Bedeutung der Leseflüssigkeit in der Fremdsprache}

Über die Bedeutung und Entwicklung der Leseflüssigkeit in der Fremdsprache (im Folgenden L2) ist vergleichsweise wenig bekannt. Zwar werden in der Literatur zur Leseflüssigkeit in der L2 die oben genannten vier Dimensionen der Leseflüssigkeit (Lesegenauigkeit, Automatisierung, Lesegeschwindigkeit, Prosodie) diskutiert, allerdings kommen diesen Dimensionen für das Lesen in der L2 teils andere Funktionen und Bedeutungen zu. Die Leseflüssigkeit ist in der L2 bedeutsam: Lesende können durch flüssiges Lesen ein größeres Maß an L2-Input bewältigen, was der Erweiterung des Wortschatzes in der Fremdsprache dient (vgl. Grabe 2009: 290; Reynolds 2014). Evident ist auch, dass die Entwicklung der Leseflüssigkeit in der Fremdsprache sich von der in der L1 unterscheidet und Lesende vor noch größere Herausforderungen stellt. 
Zum einen hängt die Entwicklung der Leseflüssigkeit in der Fremdsprache maßgeblich vom Alter der Lernenden ab, die beim Leseerwerb in der L2 meist älter sind als zur Zeit des L1-Leseerwerbs und daher andere kognitive Voraussetzung haben. Zum anderen hängt die L2-Leseflüssigkeit von den Lesefertigkeiten in der L1 und der Beschaffenheit der Fremdsprache ab, die sich linguistisch mehr oder weniger von der Erstsprache unterscheiden kann (Grabe 2009: 293). Differenzielle Transfereffekte zwischen L1 und L2 konnten Pasquarella et al. (2015) in einer Studie mit Gruppen von Erstklässler/innen, die sprachlich einen spanischen oder chinesischen Hintergrund hatten und nun in Englisch (L2) Lesen lernten, zeigen. So gab es nur in der Gruppe der Spanisch sprechenden Kinder einen Zusammenhang zwischen deren Dekodierfertigkeit in der L1 und ihrer Dekodierfertigkeit in der L2. Bezogen auf die Leseflüssigkeit hingegen fand sich ein Zusammenhang zwischen der Leseflüssigkeit in der L1 und der L2 in beiden Gruppen, was nahelegt, dass Leseflüssigkeit von einer Sprache zur nächsten übertragbar (script universal) ist. In punkto Dekodierfertigkeit sind nur dann Transfereffekte zu erwarten, wenn zwischen den Sprachen strukturelle Ähnlichkeiten bestehen und genutzt werden können.

Für die L1 wurde nach dem Simple View of Reading postuliert, dass die Leseflüssigkeit neben der Dekodierfertigkeit und dem Sprachverständnis zum Leseverständnis beiträgt. Betrachtet man die Situation von L2-Lesenden, so lässt sich wie folgt argumentieren: Zum einen kann im L2-Leseerwerb die akkurate und automatische Worterkennung nicht immer erwartet werden, was u. a. auf fehlende lexikalische Repräsentationen (Wortschatz) zurückzuführen ist. Dieser Fall tritt dann ein, wenn ein Wort in der Fremdsprache gelesen wird, der Lesende aber noch nicht über dieses Vokabular verfügt. Sinngebendes, expressives Lesen (Prosodie) ist in einer solchen Situation schwer möglich (vgl. Grabe 2009: 291). Zudem verfügen in der Fremdsprache wenig geübte Leser/innen meist über weniger entwickelte Sprachkenntnisse in der L2, was das Leseverständnis zusätzlich erschwert (vgl. Marx/Stanat 2012). Eine weitere Variable, die die Leseflüssigkeit in der L2 maßgeblich beeinflusst, ist daher, inwieweit die Fremdsprache bereits flüssig gesprochen werden kann (speaking fluency, Grabe 2009: 294). Während diese bei der Erfassung der Leseflüssigkeit in der L1 vorausgesetzt wird, kommt es beim lauten Lesen in der Fremdsprache oft zu Schwierigkeiten mit der Aussprache und Verständlichkeit. Daher zeigt sich in der Praxis, besonders am Anfang des Leseerwerbs in der L2, ein gewisser "trade-off" zwischen Lesegenauigkeit und Lesegeschwindigkeit: der Fokus der Lesenden liegt auf der Lesegenauigkeit und auf der Aussprache der einzelnen Wörter, wodurch die Lesegeschwindigkeit zurück bleibt.

In der Literatur finden sich zunehmend Studien, die die Leseprozesse in der Zweitsprache, insbesondere der Zweitsprache Englisch, untersuchen. Streng genommen unterscheiden sich Populationen von Lernenden, die Englisch als Zweitsprache (English as a second language, ESL) erwerben, und Gruppen von Lernenden, die Englisch als Fremdsprache (English as a foreign language, EFL) strukturiert im schulischen Fremdsprachenunterricht erlernen. Dennoch können Parallelen zwischen den beiden Gruppen in Bezug auf die linguistischen Kompetenzen gezogen werden: geringeres Sprachverständnis und Vokabular in der L2 verglichen mit der L1, geringere Dekodierfertigkeiten und somit auch eine verringerte Leseflüssigkeit.

Crosson/Lesaux (2010) zeigten in einer Studie mit Fünftklässler/innen aus Familien mit Migrationshintergrund, die als L1 Spanisch sprachen und über wenige Sprachkenntnisse in Englisch (L2) verfügten, dass das Leseverständnis in der L2 auch in dieser Gruppe wesentlich von der Leseflüssigkeit abhing. Dieser Einfluss bestand sogar über 
die Einschränkungen durch die wenig entwickelten Sprachkenntnisse hinaus. Eine differenzierte Analyse der Ergebnisse zeigte, dass starke Leseflüssigkeit besonders dann mit gutem Leseverständnis assoziiert war, wenn die Schüler/innen über bessere Sprachkenntnisse verfügten. Das heißt, bei Leser/innen, die schon über besser entwickelte Sprachkenntnisse in der L2 verfügten, profitierte das Leseverständnis besonders von einer größeren Textleseflüssigkeit (vgl. Yovanoff et al. 2005). Ermutigend sind auch Befunde aus Studien wie die von Lipka/Siegel (2012), die zeigen, dass Schüler/innen in Bezug auf das Leseverständnis in der L2 ein ähnliches Niveau erreichen können, wie Leser/innen in der L1.

Auch zur Förderung der Leseflüssigkeit in der L2 existieren fast keine empirischen Befunde ${ }^{1}$ oder theoretischen Überlegungen. Grabe (2009) empfiehlt, dass das Flüssigkeitstraining besonders zu Anfang des L2-Leseerwerbs nicht durch einen zu starken Fokus auf die Lesegenauigkeit behindert werden sollte. Gerade im Fremdsprachenunterricht besteht aber oft ein Widerstreben, lautes Lesen zu üben und lautes Lesen erfassend zu bewerten, da Lernende Schwierigkeit beim Lesen und bei der Aussprache zeigen. Gemäß Grabe (2009: 310) weisen allerdings die wenigen Befunde, die es zur L2-Leseflüssigkeit gibt, darauf hin, dass mit lautem Lesen geübt werden sollte, um Leseflüssigkeit und letztlich damit auch das L2-Leseverständnis zu schulen. Aus den Überlegungen zur Förderung der Leseflüssigkeit in der L1 kann geschlossen werden, dass besonders die Assisted-Reading-Lautleseverfahren für den Aufbau der Leseflüssigkeit in der L2 geeignet scheinen. Laut Segalowitz/Hulstijn (2005, zitiert in Grabe 2009: 303) kommt es beim Leseerwerb darauf an, dass Lernende mehrfach die gleichen Zuordnungen (mappings) erfahren müssen, um in der Fremdsprache Automatisierung und Flüssigkeit zu erwerben. Daher ist es wichtig, dass unbekannte Wörter/Phrasen in der Fremdsprache korrekt und konsistent gelesen und verstanden werden.

Zusammenfassend kann festgehalten werden, dass Leseflüssigkeit eine essenzielle Komponente der Lesekompetenz darstellt, die maßgebend zum Textverständnis beiträgt. Sie ist nicht nur in der Erst-, sondern auch in der Fremdsprache von Bedeutung, da sie das L2-Leseverständnis über die Einschränkungen durch wenig entwickelte Sprachkenntnisse hinaus beeinflusst. Da es sich bei der Leseflüssigkeit um ein stabiles Konstrukt handelt, sollten Schul- und Fremdsprachenlehrkräfte auf die Förderung der Leseflüssigkeit ein besonderes Augenmerk legen.

\subsection{Mehrsprachiges Lesetheater zur Förderung der Leseflüssigkeit}

Zur Förderung der Leseflüssigkeit eignen sich wie oben dargestellt Lautleseverfahren, wie z. B. Lesetheater, die gut mit motivationsfördernden Maßnahmen kombiniert werden können (siehe 1.4, vgl. Villinger et al. 2010: 167). Durch die Arbeit mit der Lautlesemethode Lesetheater wird besonders das gestaltende Lesen mithilfe des Einsatzes von Gestik und Mimik unterstützt (vgl. Teil B, Kap. 3). Ziel der neuen Fördermethode

1 Grabe (2009: 304) nennt als Ausnahme drei Arbeiten von Taguchi et al. (Taguchi 1997; Taguchi/Gorsuch 2002; Taguchi et al. 2004), die Methoden zur Förderung der L2-Leseflüssigkeit untersuchten. Allerdings wurden ausschließlich kleine Gruppen von Universitätsstudierenden beforscht, und es wurde nicht dokumentiert, ob erzielte Effekte des Leseflüssigkeitstrainings nach Beenden der Intervention erhalten werden konnten. 
Mehrsprachiges Lesetheater ist es, die Leseflüssigkeit sprachübergreifend zu trainieren, um positive Effekte auf das Textverständnis der Schüler/innen in der L1, L2 und gegebenenfalls L3 zu erzielen. Dabei werden zum einen Transfereffekte über die L1 und L2 (vgl. Pasquarella et al. 2015) und zum anderen Synergien zwischen den Sprachenfächern genutzt. Im Folgenden wird dargestellt, wie aus der Zusammenfassung des Forschungsstandes zur Leseflüssigkeit drei zentrale Aspekte abgeleitet und in der neuen Fördermethode Mehrsprachiges Lesetheater umgesetzt werden:

(1) Training der Leseflüssigkeit: Im Verlauf der Arbeit mit den Lesetheaterszenen wird die Leseflüssigkeit gleich in mehreren Arbeitsphasen trainiert. Dabei werden alle der vier oben genannten Dimensionen der Leseflüssigkeit angesprochen. Anfangs geht es um das genaue Dekodieren. Unbekannte Wörter müssen zunächst entschlüsselt werden, denn die Schüler/innen kennen die Bedeutung von einzelnen Wörtern oder Phrasen eventuell nicht und müssen sich diese zuerst erschließen. Verständnisschwierigkeiten, die u. a. durch unbekannte Wörter in den fremdsprachlichen Passagen entstehen, müssen bereinigt werden. Durch das nun folgende, mehrfache halblaute Lesen wird an der Automatisierung der Dekodierprozesse gearbeitet. Während der Unterrichtsbeobachtungen im Rahmen der formativen Evaluation des Unterrichtdesigns (vgl. Teil A, Kap. 1) zeigte sich, dass die Arbeitsgruppen ihre Szene zwischen 7- bis 15-mal (halb)laut lesen, bevor das Lesetheaterstück aufgeführt wird. In diesen Arbeitsphasen können zur intensiven Förderung der Leseflüssigkeit weitere Lautleseverfahren, insbesondere assisted reading, das sich für das Training der Leseflüssigkeit in der L2, L3 etc. eignet, eingesetzt werden. Aus der Lesegenauigkeit und der Automatisierung der Dekodierprozesse ergibt sich, wie oben argumentiert, die Lesegeschwindigkeit. An der vierten Dimension der Leseflüssigkeit, der Prosodie, arbeiten die Schüler/innen ebenfalls während des wiederholten Übens des Lautlesens. Die Schüler/innen wissen, dass gestaltendes, ausdrucksstarkes Lesen das Ziel ist, und so machen sie sich gegebenenfalls Notizen zur Betonung und arbeiten gemeinsam am sinnerfassenden Lesen. Dazu erhalten sie konstruktives Feedback von der Lehrperson und den anderen Mitschüler/innen, was besonders die Gestaltung des Lesens und eine verständliche Aussprache unterstützt.

(2) Fremdsprachliche Unterstützung: Durch die intensive Auseinandersetzung mit den fremdsprachlichen Passagen wird am Sprachverständnis in der/den Fremdsprache/n gearbeitet, was sich wiederum positiv auf das Textverstehen auswirkt. Neue, unbekannte Vokabeln werden während der Einführung in das Lesetheater besprochen. Auch die Aussprache einzelner Wörter und Phrasen muss unter Umständen geklärt werden, dabei helfen Audiofiles und Wörterbücher. Neben den Audiofiles stehen den Schüler/innen weitere Lautlesemodelle zur Verfügung: Die Lehrperson liest zur Einführung in das Lesetheater laut und mehrsprachig vor, Schüler/innen erhalten kontinuierlich Feedback zu ihrem Lesen von der Lehrperson und von ihren Mitschüler/innen.

(3) Die Passung des Lesetheatertexts an das sprachliche Niveau der Klasse nimmt in der Fördermaßnahme einen großen Stellenwert ein: Sinnerfassendes Lesen und, damit verbunden, Textverständnis können nur stattfinden, wenn der Text mit einer gewissen Lesegeschwindigkeit gelesen werden kann (vgl. Rosebrock et al. 2016: 62). Daher müssen die Texte dem Schul- und Fremdsprachenniveau der (meist heterogenen Klassen) angemessen sein, wobei besonders die limitierten Fremdsprachenkenntnisse berück- 
sichtigt werden müssen. Die Auswahl der Texte stellt die Lehrkraft daher vor besondere Herausforderungen. Hilfreich ist es deshalb, wenn sich die Lehrperson vor Beginn der Arbeit mit der Lesefördermaßnahme einen Eindruck von der Leseflüssigkeit der Schüler/innen in den verwendeten Sprachen verschafft.

\subsubsection{Die Erfassung der Leseflüssigkeit}

Leseflüssigkeit wird in der Regel über die Anzahl korrekt gelesener Wörter in einem vorgegebenen Zeitintervall erhoben. Dabei unterscheiden sich Wort- und Textleseverfahren. Im Rahmen eines Wortleseverfahrens werden Listen, bestehend aus voneinander unabhängigen Wörtern, laut vorgelesen. Bei Textleseverfahren wird hingegen ein zusammenhängender Text gelesen. Der oben genannte Zusammenhang zwischen Leseflüssigkeit und Textverständnis ist stärker, wenn Leseflüssigkeit mit einem Textleseverfahren erfasst wird (vgl. Jenkins et al. 2003). Zu Beginn der MELT-Hauptstudie wurde die Leseflüssigkeit der Schüler/innen in den im Projekt verwendeten Sprachen (mit Ausnahme der Migrationssprachen) erfasst. Im Deutschen wurde die Leseflüssigkeit der Schüler/innen in allen Partnerschulen mit der „Lernfortschrittsdiagnostik Lesen“ (LDL, Walter 2010) gemessen. Die Messung der Leseflüssigkeit im Englischen und Französischen orientierte sich streng an diesem Verfahren, setzte aber entsprechend englische bzw. französische Texte ein. Für Forschungszwecke wurde zusätzlich anhand von fünf Verständnisfragen, die für jeden Lesetext entworfen wurden, überprüft, ob die Schüler/innen das Gelesene verstanden haben. Für die Probe liest jede/r Schüler/in der Lehrperson eine Minute lang einen unbekannten Text laut vor und beantwortet im Anschluss an das Lesen die jeweiligen Verständnisfragen. ${ }^{2}$ Protokolliert werden Lesefehler und die in einer Minute insgesamt gelesenen Wörter. Bei der Auswertung werden zunächst die Anzahl der gelesenen Wörter und die Anzahl der Fehler ermittelt. Als falsch gelesen gelten Wörter, die nicht korrekt ausgesprochen oder verlängert bzw. verkürzt wurden. Auch Wörter, die durch andere Wörter ersetzt, eingefügt oder ausgelassen wurden, sowie Wörter, die nicht innerhalb von drei Sekunden gelesen werden konnten, sondern von der Lehrperson vorgegeben wurden, werden als falsch gewertet. Selbstkorrekturen gelten als richtig gelesen, ebenso wie Wörter, die durch einen Sprachfehler konsistent undeutlich bzw. falsch ausgesprochen werden. Aus diesen Werten wird die Anzahl der in einer Minute korrekt gelesenen Wörter (RWM-Wert) sowie der Prozentsatz der falsch gelesenen Wörter berechnet.

Dieses Verfahren unterstützt die Lehrperson darin, genau wahrzunehmen, welche/r Schüler/in fehlerhaft und stockend und wer genau, aber viel zu langsam und ausdruckslos liest. Wird die Probe regelmäßig durchgeführt und dokumentiert, kann die Lehrperson aufgrund des RWM-Wertes die Entwicklung der Lesegeschwindigkeit, d. h. den Lernverlauf, sehr genau beobachten (vgl. Walter 2010: 7). In der MELT-Hauptstudie wurden diese Werte verwendet, um die Leseleistung der Kooperationsklassen zu

2 Für das MELT-Forschungsprojekt wurden Audioaufnahmen vorgenommen, um die Intonationsleistungen in den verschiedenen Sprachen analysieren zu können. Die Ergebnisse werden in weiteren Publikationen dokumentiert. 
Beginn der Hauptstudie zu beschreiben ${ }^{3}$ (vgl. Teil A, Kap. 2) und um für das Niveau der Klasse geeignete Lesetheatertexte zu entwerfen und auszuwählen.

Zur vollständigen Erfassung der Leseflüssigkeit gehört des Weiteren die Analyse der vierten Dimension der Leseflüssigkeit, der Prosodie: der expressive Ausdruck, die angemessene Phrasierung, die Akzentuierung sowie die Intonation und Pausensetzung. Um die Lernentwicklung der Schüler/innen in dieser Dimension besser einschätzen zu können, ist die Oral Reading Fluency Scale von Pinnell et al. (1995) ein hilfreiches Instrument.

Tabelle 1 stellt zusammenfassend dar, wie Aspekte zur kognitiven Förderung der Leseflüssigkeit im Mehrsprachigen Lesetheater umgesetzt werden.

Tabelle 1: Die Förderung der Leseflüssigkeit im Mehrsprachigen Lesetheater

\section{Förderung der Lesefluissigkeit allgemein (Dimensionen nach Rosebrock et al. 2016)}

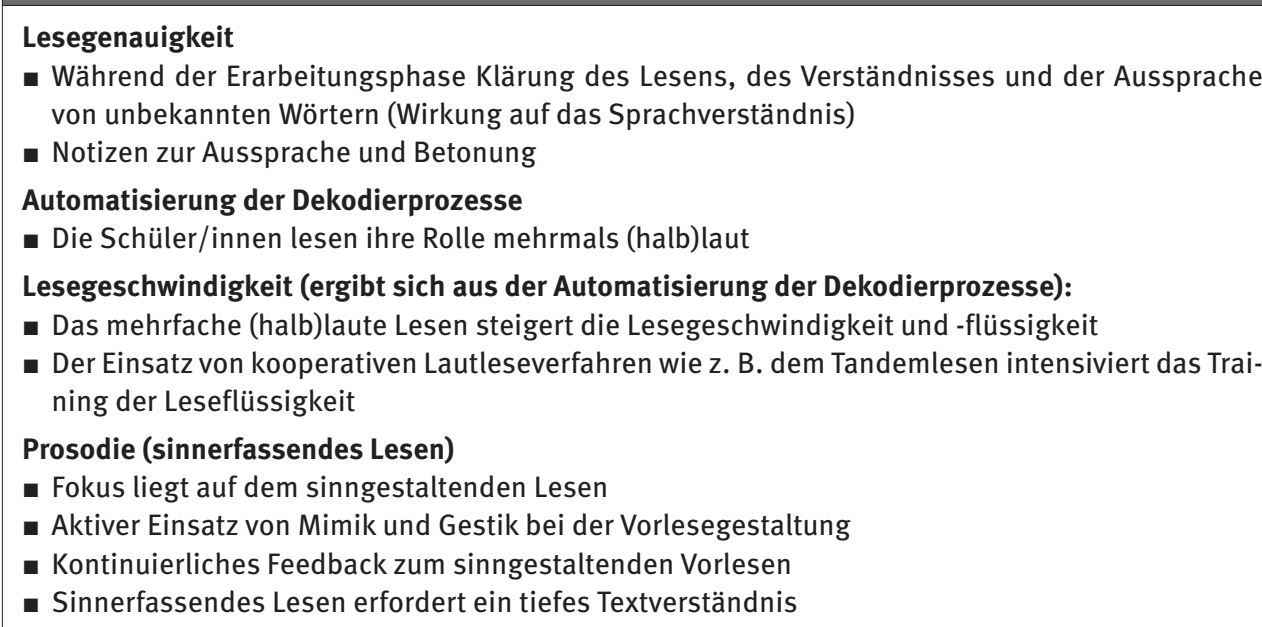

2. Unterstuitzung hinsichtlich Aussprache und Wortschatz in der Fremdsprache

- Einführung neuer Vokabeln, Klärung ihrer Bedeutung und Aussprache

- Audiofiles zur Selbstkontrolle in Bezug auf die korrekte Aussprache

- Einsatz von Lautleseverfahren (insbesondere assisted-reading) zum Training der Leseflüssigkeit in $\mathrm{der} /$ den Fremdsprache/n

3. Erfassung der Leseflüssigkeit zur Passung des Lesetheatertexts

- Erfassung der Leseflüssigkeit mit einer Lautleseprobe, um einen Eindruck vom sprachlichen $\mathrm{Ni}$ veau der Klasse zu gewinnen

- Auswahl von Texten, die von einem Großteil der Klasse mit einer Lesegeschwindigkeit von mindestens 100 Wörtern pro Minute gelesen werden können

- Eine gute Passung der Lerngegenmaterialien (Lesetheatertexte) an das sprachliche Niveau der Klasse fördert das Kompetenzerleben der Schüler/innen

3 In weiteren Publikationen wird auf die Entwicklung der Leseflüssigkeit in den schulisch geförderten Sprachen in den unterschiedlichen Anwendungskontexten eingegangen. 
Neben der Förderung der Leseflüssigkeit setzt sich Mehrsprachiges Lesetheater auch zum Ziel, die Lesemotivation der Schüler/innen zu fördern. Im Folgenden werden die theoretischen Grundlagen zur Lesemotivation und zu deren Bedeutung in der L1 und L2 besprochen, bevor die Umsetzung der motivationsfördernden Maßnahmen im Design dargestellt wird.

\subsection{Theoretische Grundlagen der Förderdimension Lesemotivation}

Lesemotivation bezeichnet die Bereitschaft einer Person, Leseaktivitäten zu initiieren (Möller/Schiefele 2004). Zahlreiche Untersuchungen belegen einen positiven Zusammenhang zwischen Lesemotivation und Lesekompetenz (vgl. Baker/Wigfield 1999; Gottfried 1990; Möller/Schiefele 2004; Unrau/Schlackmann 2006; De Naeghel et al. 2012). Dies gilt insbesondere für die intrinsische Lesemotivation (vgl. Baker/Wigfield 1999; Becker et al. 2010; Wang/Guthrie 2004), während extrinsische Lesemotivation kaum Aussagekraft bezüglich der Lesehäufigkeit und der Lesekompetenz besitzt (z. B. Becker et al. 2010; Wang/Guthrie 2004). Für die Förderung ist auch der Zusammenhang zwischen der intrinsischen Lesemotivation und der Lesehäufigkeit (z. B. Becker et al. 2010; Wang/Guthrie 2004) bedeutsam. Besonders intrinsisch motivierte Schüler/innen lesen häufiger in und außerhalb der Schule und verfügen über besser entwickelte Lesekompetenzen. Stärker ausgeprägte Motivation führt also zu vermehrter Lesepraxis, und Lesen aus eigenem Antrieb geht einher mit einer tieferen Verarbeitung des Gelesenen (vgl. auch Pfost et al. 2010; McElvany et al. 2008). Wichtig dabei ist, dass es sich um einen wechselseitigen Einfluss handelt. So argumentierten z. B. McElvany et al. (2008), dass Schüler/innen motiviert sind zu lesen, weil sie über eine ausreichende oder sogar gute Lesekompetenz verfügen. Pfost et al. (2010) zeigten, dass Schüler/innen mit besserer Lesekompetenz auch mehr außerschulische Leseaktivitäten zeigen.

Leider nimmt die Lesemotivation im Laufe der Leseentwicklung ab. Dies wurde im deutschsprachigen Raum während des Übergangs von der 2. zur 3. Klasse (vgl. Marcoulides et al. 2008; McElvany et al. 2008) sowie zur Zeit des Übergangs von der 6. zur 7. Klasse (vgl. Retelsdorf/Möller 2008; Streblow et al. 2012) dokumentiert, wobei der spätere Abfall bei Schüler/innen der Real- und Hauptschulen besonders ausgeprägt ist (vgl. Retelsdorf/Möller 2008). Für die Abnahme der Lesemotivation werden unterschiedliche Gründe genannt. Zum einen differenzieren sich bei Schüler/innen dieser Altersgruppe Interessen heraus, oder sie spezialisieren sich auf bestimmte Tätigkeiten, wobei zunehmend außerschulische Aktivitäten interessant für sie werden. Zum anderen verändert sich in dieser Phase das Kompetenzerleben der Schüler/innen, was sich auf deren Motivation zu lesen auswirkt (vgl. McElvany et al. 2008; Baumert et al. 2001). Da eine Abnahme in der Lesemotivation aufgrund des oben geschilderten $\mathrm{Zu}$ sammenhangs zu Beeinträchtigungen in der Lesekompetenz führt, sollten speziell in diesen Altersgruppen motivationale Aspekte bei der Gestaltung von Lernumgebungen und Maßnahmen zur Förderung der Lesekompetenz dringend berücksichtigt werden.

In der Regel wird die Förderung der Lesemotivation mit dem Training von Lesestrategien kombiniert, indem motivationsfördernde Maßnahmen in das Training der Lesekompetenz eingebaut werden (vgl. Guthrie et al. 2004, 2007; Rosebrock et al. 2016: 
123ff.; Streblow et al. 2007, 2012; Villinger et al. 2010). Für die Konzeption solcher Maßnahmen wird oft die Selbstbestimmungstheorie (Deci/Ryan 1993, 2000; Ryan/ Deci 2000) herangezogen. Laut Deci/Ryan (1993: 229f.) wirken drei angeborene psychologische Grundbedürfnisse (basic needs) gewissermaßen als Motor für das ständige Streben des Menschen nach persönlicher Entwicklung und Wohlbefinden: Das Bedürfnis nach Autonomie, das Bedürfnis, sich selbst als kompetent oder wirksam zu erfahren, und das Bedürfnis, sich sozial eingebunden zu fühlen. Auf dem Hintergrund empirischer Befunde verdeutlichen Deci/Ryan (ebd. 230ff.) die Relevanz der psychologischen Grundbedürfnisse für die pädagogische Praxis: Autonomie und Kompetenz führen zur menschlichen Bereitschaft, etwas zu leisten. Das Bedürfnis, sozial dazuzugehören, ermöglicht u. a. die Identifikation mit von außen vorgegebenen Zielen, z. B. durch Eltern und Lehrpersonen (ebd. 230ff.). Erleben sich Schüler/innen als autonom, kompetent und sozial eingebunden, ist dies mit positiven Gefühlen verbunden, was wiederum das Verhalten verstärkt.

Streblow et al. (2012) untersuchten die Wirkung eines Leseförderprogramms, das das Training von Lesestrategien mit motivationsfördernden Maßnahmen verknüpfte (LeKoLeMo: Programm zur Förderung der LeseKompetenz und LeseMotivation). Das Leseförderprogramm wurde über einen Zeitraum von drei Wochen in einer Gruppe Siebtklässler/innen angewandt, und es zeigte sich ein kurzfristiger, positiver Effekt auf die intrinsische Lesemotivation der Schüler/innen. Allerdings beruhte dieser Effekt zum Teil auf einer Senkung der Motivationswerte der Kontrollgruppe, die kein Training erhielt. Zudem war dieser positive Effekt nicht nachhaltig, denn bereits zwei Monaten nach Ende der Intervention wurden keine Unterschiede mehr zwischen den Gruppen gefunden.

$\mathrm{Zu}$ ähnlichen Ergebnissen kommen Villinger et al. (2010) mit jüngeren Schüler/innen. In der LiFuS-Studie (Lesen in Familie und Schule) wurde untersucht, wie sich drei unterschiedliche Fördermaßnahmen auf die Lesemotivation und das Textverständnis von Viertklässler/innen auswirken. Es zeigte sich wie bei Streblow et al. (2012), dass die Interventionen zwar einen positiven Effekt auf die Lesemotivation hatten, der sich aber nach Beendigung der Maßnahme nicht aufrechterhielt. Auch bei der Kontrollgruppe, die keine Intervention erhielt, nahm die Lesemotivation stetig ab und dies bereits in der 4. Klassenstufe. Zusammengenommen zeigen diese Studien, dass sich der Einbezug von motivationalen Komponenten in das Design von Lesefördermaßnahmen bewährt, aber zu keinen nachhaltigen Effekten führt.

\subsection{Die Bedeutung der Lesemotivation in der Fremdsprache}

Obwohl die L2-Motivationsforschung zunehmend Aufmerksamkeit erfährt (vgl. Boo et al. 2015), befassen sich sehr wenige empirische Studien spezifisch mit der Motivation für das Lesen in der Fremdsprache. Netten et al. (2011) untersuchten, welche Faktoren das Leseverständnis von Schüler/innen, die in der Erst- bzw. Zweitsprache lesen, beeinflussen. Bezüglich der Lesemotivation zeigten beide Gruppen ähnliche Werte. Auch zeigte sich in beiden Gruppen ein positiver Zusammenhang zwischen Lesemotivation und Leseverständnis. Ebenso konnte eine Untersuchung von Lin et al. (2012) mit chinesischsprachigen Fünftklässler/innen aus Hong Kong, die Englisch als Fremdsprache in der Schule lernten, einen Zusammenhang zwischen Lesemotivation und 
Textverständnis in der L2 belegen. Interessant ist, dass die Schüler/innen, verglichen mit der L2-Lesemotivation, wesentlich mehr intrinsische Lesemotivation in der Erstsprache aufwiesen. Demnach lesen Lernende eher in der etablierten Erstsprache, in der weniger sprachliche Hürden erfahren werden. Zudem steht hier Lesestoff meist leichter und in einer größeren Vielfalt zur Verfügung. Außerdem dient Lesen oft dem Erholungszweck, oder es wird gelesen, um sich Wissen anzueignen. In der Fremdsprache hingegen bedarf es eines hohen Maßes an Motivation, um sich trotz sprachlicher Hürden weiterhin mit dem Lesen und dem Lesestoff zu verbinden und wiederholt zu üben (vgl. Grabe 2009: 135). In der Studie von Lin et al. war für das L2-Leseverständnis besonders die instrumentelle Dimension der Lesemotivation von Bedeutung. Diese reflektiert u. a. ein Verständnis von den Vorteilen des Fremdsprachenerwerbs: Je bewusster sich Schüler/innen der positiven Aspekte und des Nutzens der Lesekompetenz in der Fremdsprache waren, desto besser war ihr L2-Leseverständnis.

Diese Befunde implizieren für die Förderung der Lesemotivation in der Fremdsprache Folgendes: Intrinsische Motivation, die in der L1 äußerst wichtig ist, ist in der Fremdsprache vielleicht weniger bedeutend. Ferner ist es ratsam, Lernenden die Bedeutung des Lesens in der Fremdsprache zu verdeutlichen. Zwar ist bislang noch ungeklärt, wie genau die Lesemotivation in der L2 gefördert werden sollte, aber es ist anzunehmen, dass sich auch hier der Einbezug von motivationsförderlichen Maßnahmen bewähren kann. So bezieht sich auch Grabe (2009: 191) in seiner Diskussion der Literatur zur Lesemotivation für den (Fremd-)Sprachenunterricht auf die Selbstbestimmungstheorie nach Deci/Ryan: (1) Unterricht sollte den Lernenden erfolgreiche Lernerfahrungen ermöglichen. Das geschieht, indem die Lehrperson zuvor die Fertigkeiten der Schüler/innen richtig einschätzt und das Niveau der Unterrichtsaufgaben so ansetzt, dass Schüler/innen Lernerfolge erzielen und auch wahrnehmen. (2) Zudem sollte die Autonomie der Schüler/innen unterstützt und (3) die Zusammenarbeit zwischen den Lernenden gestärkt werden.

\subsection{Mehrsprachiges Lesetheater zur Förderung der Lesemotivation}

Die Selbstbestimmungstheorie nach Deci/Ryan (1993; 2000) diente im Entwicklungsund Forschungsprozess von MELT als theoretischer Orientierungsrahmen, um die motivationalen Bedingungen im Lehr-Lern-Prozess günstig zu gestalten. Bezogen auf die drei Grundbedürfnisse sollen sich die Schüler/innen weitgehend autonom, kompetent und sozial eingebunden erleben können, damit Mehrsprachiges Lesetheater in der Unterrichtssituation intrinsisch motivierend wirkt. Konkret wurde dies folgendermaßen im Unterrichtsdesign umgesetzt:

(1) Autonomie: Während die Schüler/innen auf die Aufführung hinarbeiten, handeln sie in unterschiedlichen Phasen autonom: In der Vorbereitung zum gestaltenden Lesen setzen sie sich selbstbestimmt mit ihrer Rolle auseinander (die sie je nach Vorgehensweise der Lehrperson gegebenenfalls sogar selbst aussuchen durften), indem sie sich selbstständig die sprachliche Gestaltung der zu lesenden Rolle erarbeiten. Die Schüler/innen können bei Bedarf auf Audiounterstützung für die fremd- und migrationssprachlichen Leseteile zurückgreifen, um die Leseflüssigkeit selbstständig zu trainieren. Im Ermessen der Lehrperson liegt, ob die Schüler/innen bei der Auswahl der Lesetheatertexte 
mitentscheiden dürfen, da ein Mitspracherecht sich günstig auf das Autonomieempfinden auswirkt.

(2) Kompetenzerleben: Die Schüler/innen erleben sich als kompetent, wenn sie ihre Rolle nach den Übungsphasen flüssig lesen können. Dies ist in Bezug auf alle verwendeten Sprachen wichtig, auch für die Schulsprache, die für zunehmend mehr Schüler/innen nicht die L1 ist. Kompetenz empfinden die Schüler/innen ebenfalls, wenn sie aufgrund der Mehrsprachigkeit der Lesetheater in der Lage sind, Szenen aus Texten zu präsentieren, deren eigenständige Erarbeitung und Lektüre für sie, besonders in der Fremdsprache, noch nicht möglich wäre. Auch in der intensiven Gruppenarbeit, wenn sich die Schüler/innen gegenseitig unterstützen und Feedback geben, können sie sich als kompetent wahrnehmen. Natürlich wirkt positives Feedback der Lehrperson hier ebenso. Wenn Migrationssprachen mit in das Lesetheater einbezogen werden, sind die Schüler/innen, die diese Sprache beherrschen, die Expert/innen und fühlen sich kompetent und wertgeschätzt.

(3) Soziale Eingebundenheit: Die Schüler/innen sind Teil einer Arbeitsgruppe, die ein gemeinsames Ziel hat, nämlich ihre Szene für das Publikum interessant zu präsentieren. Dies kann nur gelingen, wenn alle in der Gruppe ihr Bestes geben und keiner sich herausnimmt. Wenn die Schüler/innen dann ihre Szene aufführen, Applaus ertönt und positives Feedback gegeben wird, können sie sich angenommen fühlen. Soziale Eingebundenheit entsteht aber auch im literarischen Gespräch; dabei können sich die Schüler/innen miteinander austauschen und soziale Verbindungen zueinander aufbauen.

Motivationale Aspekte spielen nicht nur im mehrschrittigen Lehr-Lern-Prozess eine entscheidende Rolle, sondern auch bei der Auswahl und der Gestaltung der LehrLern-Materialien. Um die Leselust der Schüler/innen zu wecken, wurden Texte aus der Kinder- und Jugendliteratur ${ }^{4}$ ausgewählt, die die Interessen der unterschiedlichen Altersgruppen widerspiegeln und eine Identifikation mit den Protagonist/innen sowie die Herleitung eines Bezugs zur Lebenswelt der Schüler/innen zulassen. Im Sinne des Kompetenzerlebens wurde ferner auf eine angemessene Textlänge geachtet. Der Anteil sowie der Schwierigkeitsgrad der fremdsprachlichen Passagen wurde variiert, um eine gute Passung zwischen Lesetheater und Klasse zu ermöglichen. Des Weiteren standen unterschiedlich anspruchsvolle Rollen zur Verfügung, die differenziert den Kompetenzen der einzelnen Schüler/innen zugewiesen werden konnten. So wurde das Kompetenzerleben der Schüler/innen gestärkt.

Analog zur Erfassung der Leseflüssigkeit am Anfang der MELT-Hauptstudie wurde auch die Daten zur Lesemotivation der Schüler/innen mithilfe eines Fragebogens zur habituellen Lesemotivation (FHLM, Möller/Bonerad 2007) erhoben. Der FHLM erfasst die intrinsischen Komponenten der Lesemotivation als Leselust (5 Items, z. B.: „Es macht mir Spaß, Bücher zu lesen.") und Leseinteresse (5 Items, z. B.: „Ich finde Lesen interessant."). Des Weiteren wird eine eher extrinsische Komponente der Lesemotivation über fünf wettbewerbsorientierte Items erfasst (z. B.: „Wenn wir im Unterricht le-

4 Das ursprüngliche Ziel des MELT-Projekts war es, vor allem Werke aus der aktuellen Kinderund Jugendliteratur zu adaptieren. Aus Gründen des Urheberrechts musste vom Projektplan abgewichen werden und auf copyright-freie literarische Texte für die Dramatisierung zurückgegriffen werden (vgl. Teil B, Kap. 3). 
sen, versuche ich, besser zu sein als die anderen."). Die Schüler/innen beantworteten die Items auf einer vierstufigen Skala von „stimmt gar nicht“ bis „stimmt genau“. Diese Werte wurden im Rahmen der MELT-Studie hauptsächlich verwendet, um das Motivationsprofil der Kooperationsklassen zu beschreiben (vgl. Teil A, Kap. 2). Weitere Analysen der Zusammenhänge zwischen Leseflüssigkeit und -motivation in den verschiedenen Sprachen und unterschiedliche Fallanalysen zu intrinsisch oder extrinsisch motivierten Schüler/innen werden in weiteren Publikation außerhalb des Handbuchs aufgegriffen.

Abschließend wird die Umsetzung der motivationsfördernden Maßnahmen im Mehrsprachigen Lesetheater in Tabelle 2 zusammengefasst:

Tabelle 2: Motivationsfördernde Merkmale des Mehrsprachigen Lesetheaters

Förderung der Lesemotivation (nach Deci/Ryan 1993; 2000)

\section{Autonomie}

- Individuelle Auseinandersetzung mit der darzustellenden Rolle

- Selbstständiges Erarbeiten der sichtbaren und hörbaren sprachlichen Mittel des gestaltenden Vorlesens

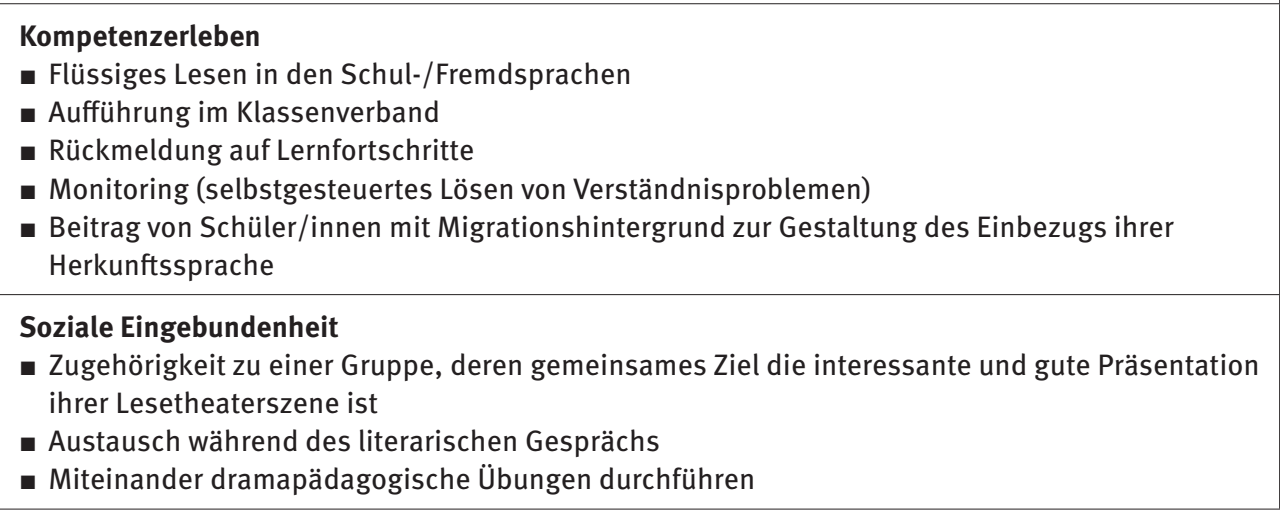

In diesem Kapitel wurde der Forschungsstand, bezogen auf die Förderdimensionen Leseflüssigkeit und -motivation, dargestellt; dabei wurde auch auf mehrsprachige Aspekte eingegangen. Diese Forschungsdesiderate fungierten während des Entwicklungs- und Forschungsprozesses als theoretischer Bezugsrahmen, um mithilfe der anwendungsorientierten DBR-Forschungsstrategie (vgl. Teil A, Kap. 1) ein in der Praxis umsetzbares Design zur mehrsprachigen Leseförderung zu gestalten. Die Befunde der Akzeptanzstudie zeigen (vgl. Teil A, Kap. 3), dass sich Mehrsprachiges Lesetheater in der Praxis als ein nutzbringendes und praktikables Unterrichtsdesign erwiesen hat. Die theoretischen Grundlagen, die in diesem Beitrag beschrieben wurden, waren auch die Grundlage für zwei Fortbildungsmodule des projektinternen Lehrertrainings, die die beiden Förderdimensionen der Lesefördermaßnahme zum Thema machten. Damit trägt die Lesefördermaßnahme MELT zu dem langsam, aber stetig wachsenden Bestand an mehrsprachigen Maßnahmen und Materialen für den Unterricht bei, der Lehrpersonen bei der Umsetzung der Mehrsprachigkeitsdidaktik unterstützt. 